\title{
The Anti-Proliferative Lichen-Compound Protolichesterinic Acid Inhibits Oxidative Phosphorylation and Is Processed via the Mercapturic Pathway in Cancer Cells
}

(ㄷ) (1) (ㄹㅇㅇ

\author{
Authors \\ Affiliations \\ 1 Faculty of Medicine, University of Iceland, Reykjavik, \\ Iceland \\ 2 Faculty of Pharmaceutical Sciences, University of Iceland, \\ Reykjavik, Iceland
}

Freyr Jóhannsson ${ }^{1}$, Paulina Cherek ${ }^{1}$, Maonian $\mathrm{Xu}^{2}$, Óttar Rolfsson ${ }^{1}$, Helga M. Ögmundsdóttir ${ }^{1}$

Key words

Protolichesterinic acid, mitochondria, cancer, metabolism, Cetraria islandica, Parmeliaceae

$\begin{array}{ll}\text { received } & \text { March 4, } 2021 \\ \text { accepted after revision } & \text { August 3, } 2021 \\ \text { published online } & \text { September 14, 2021 }\end{array}$

Bibliography

Planta Med 2022; 88: 891-898

DOI 10.1055/a-1579-6454

ISSN 0032-0943

(C) 2021. The Author(s).

This is an open access article published by Thieme under the terms of the Creative Commons Attribution-NonDerivative-NonCommercial-License, permitting copying and reproduction so long as the original work is given appropriate credit. Contents may not be used for commercial purposes, or adapted, remixed, transformed or built upon. (https://creativecommons.org/licenses/by-nc-nd/4.0/)

Georg Thieme Verlag KG, Rüdigerstraße 14,

70469 Stuttgart, Germany

\section{Correspondence}

Professor Helga M. Ögmundsdóttir

Faculty of Medicine, School of Health Sciences,

University of Iceland

Sturlugata 8, 102 Reykjavik, Iceland

Phone: + 3548918695 , Fax: + 3545254884

helgaogm@hi.is $\bigoplus \begin{aligned} & \text { Supplementary material is available under } \\ & \text { https://doi.org/10.1055/a-1579-6454 }\end{aligned}$

\section{ABSTRACT}

The lichen compound protolichesterinic acid (PA) has an antiproliferative effect against several cancer cell lines of different origin. This effect cannot be explained by the known inhibitory activity of PA against 5- and 12-lipoxygenases. The aim was therefore to search for mechanisms for the anti-proliferative activity of PA. Two cancer cell lines of different origin, both sensitive to anti-proliferative effects of PA, were selected for this study, T-47D from breast cancer and AsPC-1 from pancreatic cancer. Morphological changes were assessed by transmission electron microscopy, HPLC coupled with TOF spectrometry was used for metabolomics, mitochondrial function was measured using the Agilent Seahorse XFp Realtime ATP assay and glucose/lactate levels by radiometry. Levels of glutathione, NADP/NADPH and reactive oxygen species [ROS] were measured by luminescence. Following exposure to PA both cell lines showed structural changes in mitochondria that were in line with a measured reduction in oxidative phosphorylation and increased glycolysis. These changes were more marked in T-47D, which had poorer mitochondrial function at baseline. PA was processed and expelled from the cells via the mercapturic pathway, which consumes glutathione. Nevertheless, glutathione levels were increased after 24 hours of exposure to PA, implying enhanced synthesis. Redox balance was not much affected and ROS levels were not increased. We conclude that PA is metabolically processed and expelled from cells, leading indirectly to increased glutathione levels with minimal effects on redox balance. The most marked effect was on mitochondrial structure and metabolic function implying that effects of PA may depend on mitochondrial fitness.

\section{Introduction}

New drugs can be developed by one of two main approaches; by synthesizing tailor-made compounds to fit a proposed drug target or by screening existing compounds for a desired activity. In the latter case, the mode of action is often unknown. The aliphatic $y$ lactone protolichesterinic acid (PA, > Fig. 1) is one of the major secondary metabolites in the lichen Cetraria islandica (L.) Ach. (Parmeliaceae, Ascomycota [1,2]). This compound was described several years ago to have anti-proliferative effects against cancer 


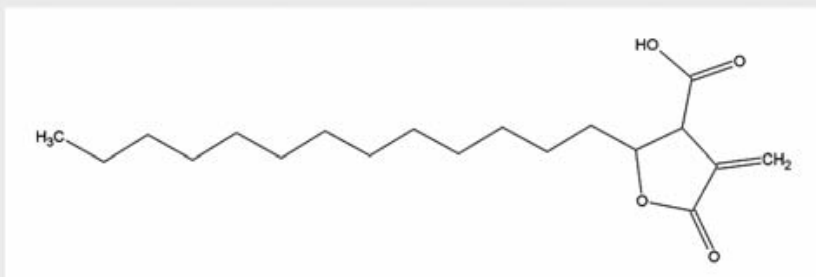

Dig. 1 Structure of the aliphatic $y$-lactone protolichesterinic acid. This was isolated from the lichen Cetraria islandica.

cells, but not against normal fibroblasts or resting lymphocytes [3-5]. PA was previously known to inhibit 5-lipoxygenase [6] and subsequently shown to also inhibit 12-lipoxygenase [7]. At that time, it had been proposed that 5- and 12-lipoxygenases (LOX) promoted carcinogenesis and cancer progression [8]. We found that PA could indeed inhibit the activity of 5 - and 12 LOX in cancer cells but this required concentrations that were much higher than those needed for anti-proliferative effects. This implies that the anti-proliferative activity of PA is not mediated by inhibition of 5and 12 LOX [9]. The structural similarity between PA and C75, a known inhibitor of fatty acid synthase (FASN) suggested another possible mode of action. Our results indicated that PA affects FASN activity in breast cancer cells that overexpress this enzyme, but not in another breast cancer cell line that does not overexpress FASN [10]. Furthermore, PA affected signalling through the ERK $1 / 2$ and AKT pathways, but these effects were context-dependent and secondary [10].

Having thus failed to identify a basic effect of PA on cells we were prompted to search for other mechanisms and effects on cellular function. PA was shown in a screening study several years ago to have inhibitory activity against HIV reverse transcriptase [11]. One frequently reported off-target effect of HIV RT inhibitors is mitochondrial toxicity, mediated by inhibition of the mitochondrial DNA polymerase $y$ [12]. In addition, our preliminary transmission electron-microscopic studies had suggested that PA might induce morphological changes in mitochondria. The aim of the current study was, therefore, to explore the effects of PA on mitochondrial structure and function, using a combined approach of transmission electron microscopy (TEM), mass spectrometry, and specific tests for effects on energy metabolism, radical oxygen species (ROS) and redox status.

\section{Results}

Two cell lines were selected for the current study based on previous results. Both are sensitive to the anti-proliferative effects of PA with almost the same $I_{50}(3.5 \mu \mathrm{g} / \mathrm{mL}$ for AsPC- 1 and $3.7 \mu \mathrm{g} /$ $\mathrm{mL}$ for T-47D) by Crystal violet survival assay or thymidine uptake $[4,9,10]$, but there were indications that they responded differently, as AsPC-1 showed less reduction in the S-phase fraction of the cell cycle. Transmission electron microscopy revealed that the two cell types had different mitochondrial morphologies in cells treated with solvent control (see - Fig. 2). In AsPC-1, most mitochondria were long and slender, whereas they were short and

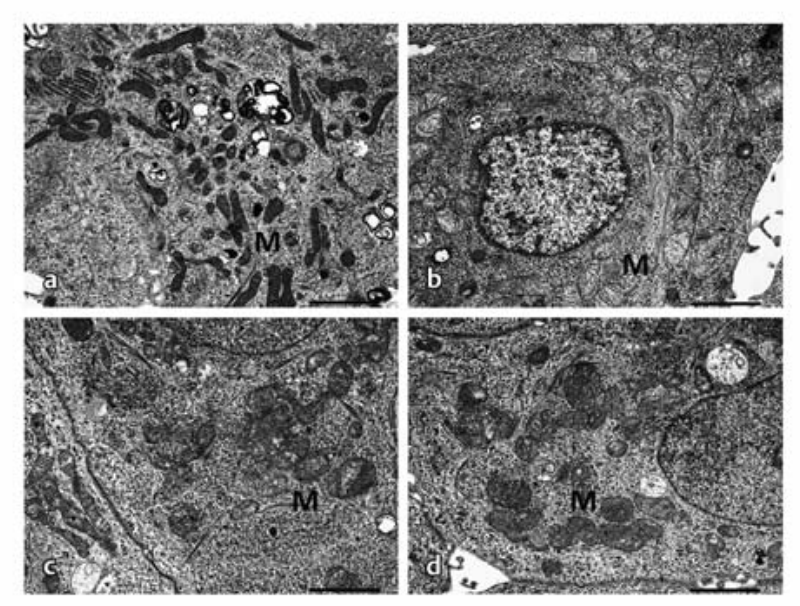

- Fig. 2 PA induces structural changes in mitochondria. a ASPC-1 solvent control; b ASPC-1 PA $5 \mu \mathrm{g} / \mathrm{mL}$; c T47-D solvent control; d T47-D PA $5 \mu \mathrm{g} / \mathrm{mL}$. Magnification: $5000 \times$, scale bars: $2.0 \mu \mathrm{m}$. Mitochondria are marked with $\mathrm{M}$.

broad in T-47D. PA induced changes in both cell lines. After exposure of T-47D to PA mitochondria appeared shorter and swollen, many had lost their inner structure, and cristae were fragmented. The effect of PA on AsPC- 1 was less marked, but in PA-treated cells short and broad mitochondria were clearly more prominent than in cells exposed only to solvent.

Having observed morphological changes in mitochondria upon treatment with PA we tested for effects on energy metabolism. Measurements of glucose uptake and lactate secretion in spent medium after 24 hours was indicative of lowered glycolytic rate, although these changes were not statistically significant (Supplementary Fig. 1S). To enhance the sensitivity of measurements and more accurately determine changes in metabolic rate, we performed metabolic flux analysis using the Seahorse technology ( $\vee$ Fig. 3). When cells were exposed to PA for 24 hours before the analysis, a clear effect was observed in both cell lines with reduced oxygen consumption and increased acidification. Comparison of untreated controls indicates that oxidative phosphorylation was already lower, and acid-producing glycolysis higher in T-47D than AsPC-1, shown by the less marked effect of adding oligomycin (uncoupling ATP production from oxygen uptake). When response was monitored during addition of PA the presence of PA

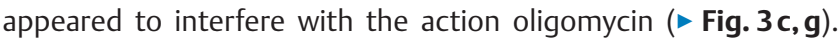
Nevertheless, rapid acidification occurred in T-47D, but not AsPC-1, which might imply already poorer mitochondrial function in T-47D and therefore greater sensitivity ( $\bullet$ Fig. $\mathbf{3 d}$, h). The differences observed in pre-treated vs. titrated response suggest that PA has not fully imparted its metabolic effect within the 100-minute timeframe of the seahorse titration assay.

To search for general metabolic effects of PA, a metabolomic screen of intracellular metabolites following PA treatment was performed. The results revealed that PA itself is processed through the mercapturic pathway, yielding glutathione conjugates that are further metabolized into cystenyl-glycine conju- 


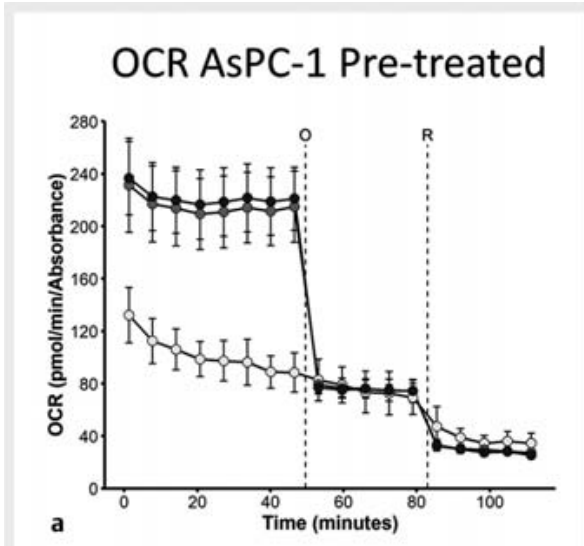

ECAR AsPC-1 Pre-treated

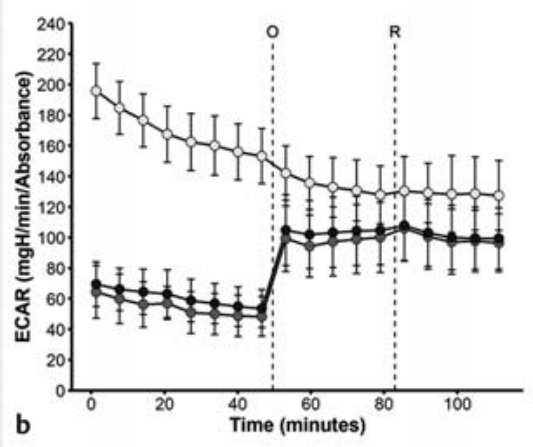

OCR T-47D Pre-treated

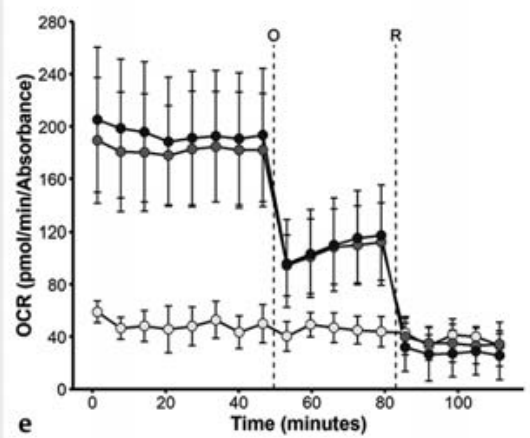

OCR AsPC-1 Titrated

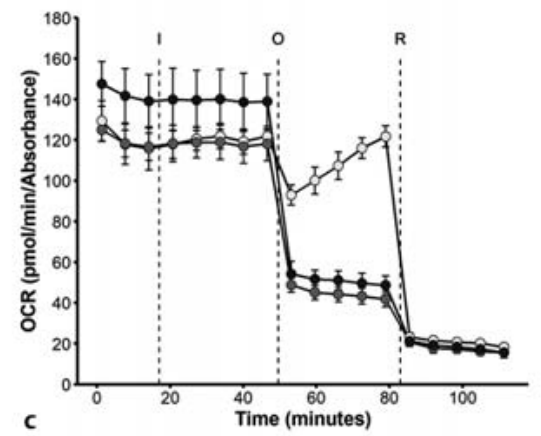

ECAR T-47D Pre-treated

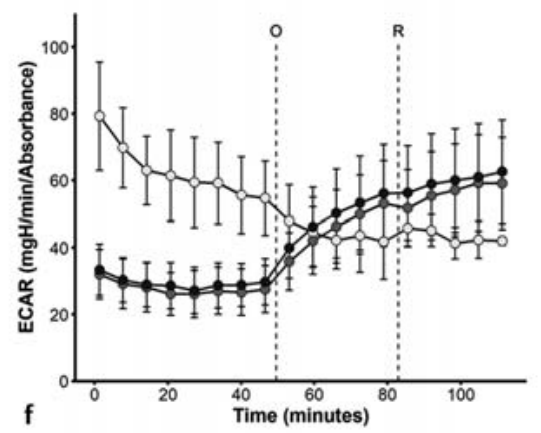

d
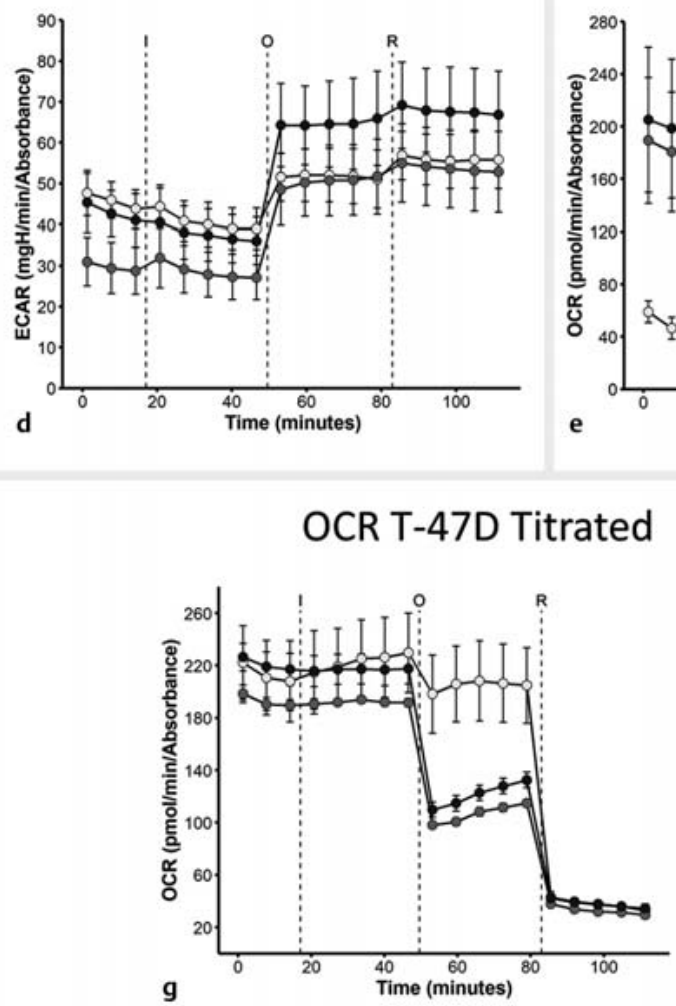

- Fig. 3 PA inhibits oxidative phosphorylation in cell models of breast and pancreatic cancer. Seahorse flux analysis showing oxygen consumption rates (OCR) and extracellular acidification rates (ECAR) of AsPC-1 and T-47D cells pretreated (a, b, e and $\mathbf{f}$ ) or titrated with PA (c, d, $\mathbf{g}$ and $\mathbf{h})$. Injections were as follows: I: Injection of PA $(0.5 \mu \mathrm{g} / \mathrm{mL})$. O: Oligomycine $(1.5 \mu \mathrm{M})$. R: Rotenone/Antimycine $(0.5 \mu \mathrm{M})$. Black is control, dark grey is solvent control $(\mathrm{EtOH})$ and light grey is PA. Each panel is representative of 4 experimental replicates $(n=4)$, each performed in technical triplicate. The error bars represent standard deviation.

gates and finally cysteine conjugates ( $\bullet$ Fig. 4 ). The same compounds were detected in the extracellular media indicating their excretion from the cell (Supplementary Fig. 2S).

At the same time, the total levels of glutathione were elevated in both cell lines, implying enhanced synthesis; see $\boldsymbol{\nabla}$ Fig. 4 . The measured increase in levels of oxidized glutathione was less marked and not significant. Other significant metabolic changes detected in the screen were a decrease in glutamate levels and in- creased levels of proline. These two changes are linked, since proline can be derived from glutamate in a biosynthetic process requiring $\mathrm{NAD}[\mathrm{P}] \mathrm{H}[13,14]$. Minor changes were observed in choline lipids, $20-50 \%$ increase in levels of lysophspatidyl choline, and $10-60 \%$ increase in carnitine. These changes were greater in T-47D and could be related to cell death, which was more marked in this cell line (Supplementary Fig. 3S). 


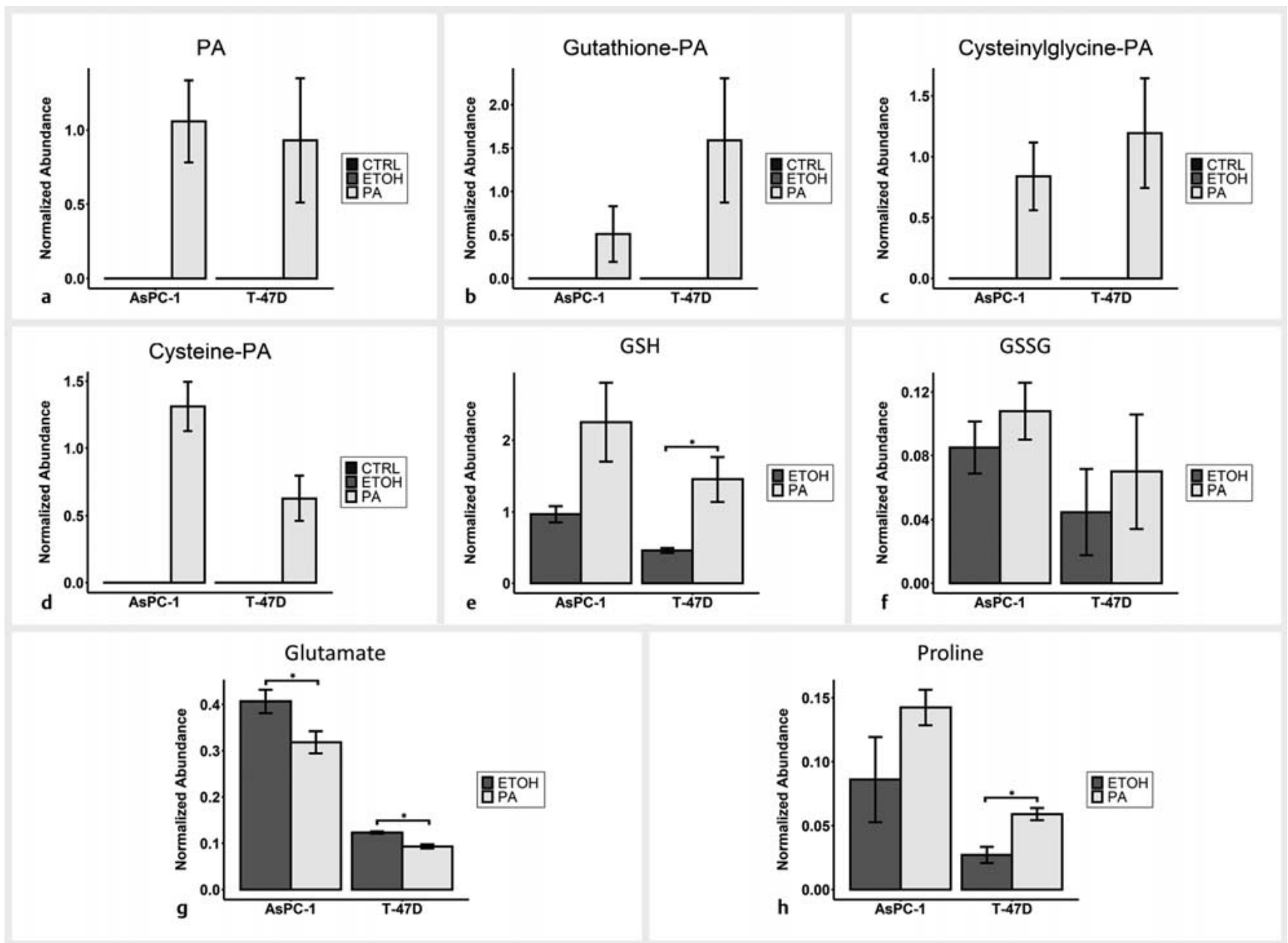

- Fig. 4 PA is conjugated to glutathione and compounds of the mercapturic pathway. LCMS analysis of intracellular metabolic extracts revealed increased abundance of PA following $24 \mathrm{~h}$ treatment (a) and PA was conjugated with glutathione (b), cystenylglycine (c) and cysteine (d). Black is control, dark grey is solvent control (EtOH) and light grey is PA. Confirmatory LCMS fragmentation spectra of these compounds are afforded in Supplementary Fig. 2S. Statistical analysis of detected LCMS features revealed differences to reduced and oxidized glutathione (e and $\mathbf{f}$ ) and glutamate $(\mathbf{g})$ and proline $(\mathbf{h})$. The error bars represent standard deviation of all measurements $(n=9)$. Brackets indicate significant differences ( $p<0.05$, Bonferroni corrected Welch's t-test).

Having found that glutathione is utilized in metabolic processing of PA, we sought to confirm how PA affected redox balance. The effect on glutathione levels was investigated further at different time points, using an enzymatic assay. The significant increase in total glutathione levels was confirmed in both cell lines after 24 hours of exposure (42\% and 57\% in AsPC-1 and T-47D. respectively), and the effect was already evident in AsPC-1 after 6 hours (37\%). Levels of oxidized glutathione were not affected after 6 hours but elevated in both cell lines after 24 hours (31\% and $28 \%$ in AsPC- 1 and T-47D. respectively) ( $\bullet$ Fig. 5).

The increased levels of glutathione were reflected in reduced levels of NADPH (to approximately $80 \%$ in both cell lines at 6 and $24 \mathrm{~h}$; Fig. 5), which is required for reduction of oxidized glutathione to regenerate the reduced form. The levels of NADP+ were also reduced and therefore the ratio NADPH/NADP+ was not significantly altered.
PA did not induce a rise in reactive oxygen species (ROS). A small but significant reduction was observed in AsPC-1 after 24 hours when a marked response was seen in the positive control with menadione (Supplementary Fig. 4S). Taken together, the results confirm changes to glutathione but the overall redox balance appeared not to be much affected.

\section{Discussion}

This study has revealed how protolichesterinic acid is metabolically processed and conjugated through the mercapturic pathway. The only other significant finding from the metabolic screen was increased production of proline from glutamate. The consumption of glutathione in the conjugation stimulated synthesis of glutathione, resulting in significantly raised levels. Redox balance was not much affected, and ROS levels were not increased. These findings were the same in both cell lines tested. The two cell 

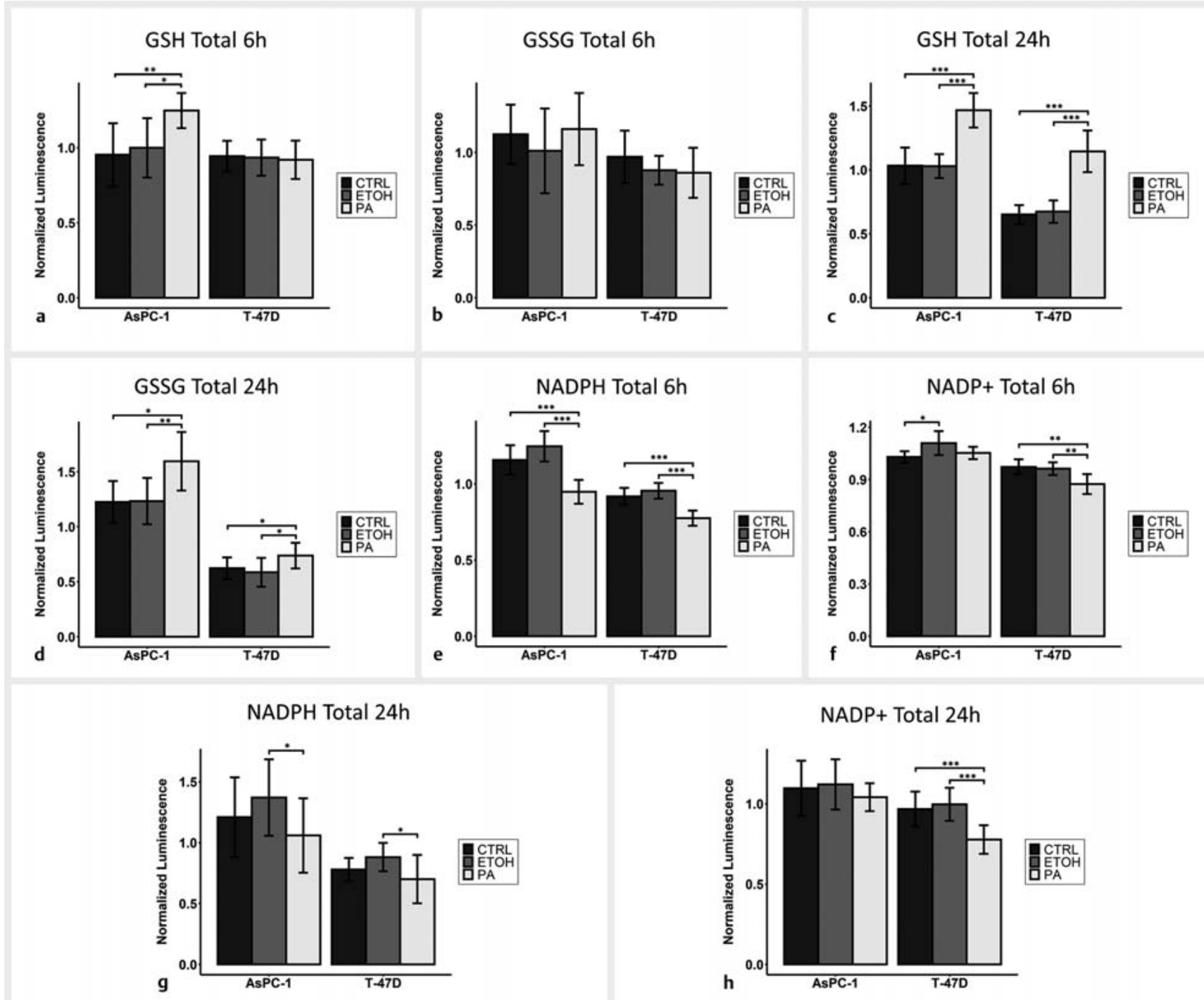

- Fig. 5 PA treatment impacts intracellular glutathione and NADPH. Enzymatic assays of intracellular glutathione and NADPH levels. The error bars represent standard deviation of all measurements $(n=9)$. Brackets indicate significant differences $(p<0.05$, Bonferroni-corrected Welch's t-test). a, b glutathione 6 h; c, d glutathione 24 h; e, f NADPH and NADP+ 6 h; g, h NADPH and NADP+ 24 h.

lines showed different mitochondrial morphology and function at base line and also differed in the effects observed on mitochondria and energy metabolism following exposure to PA, with T-47D having poorer mitochondrial function at baseline and showing more markedly reduced function in response to PA. Taken together, PA therefore seems to have two main effects on cellular metabolism. Glutathione levels are increased following conjugation that expels PA from the cell. The overall effect on redox balance is minimal. Mitochondrial structure and function are affected, with a reduction in oxidative phosphorylation and increased glycolysis with reduced flux through the TCA cycle, directing glutamate to alternative pathways.

The chemical structure of PA makes it a candidate for metabolic processing by conjugation to glutathione by Michael addition [15]. Following the first step of conjugation with glutathione, further processing takes place extracellularly. The compound is thus inactivated and expelled from the cell. As the conjugation consumes glutathione, it might be expected that its levels would decline. This was indeed the case in the study of Punganuru et al. on goniothalamin [16] that is processed in the same way. In contrast, our study showed increased levels of glutathione in PA-treated cells, indicating stimulated synthesis. The biosynthesis of glutathione subunits is regulated by the transcription factor Nrf2 [17]. Interestingly, derivatives of PA have been reported to activate Nrf2 [18].

Despite the redox balance (i.e., ratios of NADPH/NADP+ and GSH/GSSG and ROS levels) being relatively unaffected and consistent with the findings of Brisdelli et al. [3], a clear increase in intracellular glutamate and proline was observed following PA treatment. Proline is generated from glutamate in enzymatically catalysed reactions that are dependent upon NADPH. As the NADPH levels were lowered, although redox balance was relatively un- 
affected, this indicates that a change in glutamate levels drives the increase in intracellular proline. Thus, our data imply that enhanced GSH synthesis could lead to enhanced flux via the GSH synthesis pathway, thus resulting in increased glutamate levels and proline overflow. Indeed, the mercapturic pathway stimulates enhanced GSH synthesis via Nrf2 [17] transcriptional activation of glutamate cysteine ligase, the rate limiting enzyme of GSH synthesis. The increase in $\mathrm{GSH} /$ glutamate and proline might therefore be explained via Nrf2 activation. Quantitatively, however, given shutdown of oxidative phosphorylation after $24 \mathrm{~h}$, reduced flux within the TCA cycle and a build-up of TCA cycle intermediates and their precursors (i.e., glutamate) is a more likely explanation of increased levels of glutamate rather than enhanced GSH synthesis per se. Glutamate is then directed to other pathways including GSH and proline synthesis. Albeit consistent with current understanding of the mechanism of anaplerotic fuelling of the TCA cycle, a more detailed flux analysis incorporating isotopically labelled nutrient precursors would be required to confirm these flux hypotheses. Nevertheless, considering the seahorse flux analysis, $24 \mathrm{~h}$ treatment of PA clearly results in a shutdown of oxidative phosphorylation.

The role of mitochondria in cancer is now well recognized and goes much beyond the "Warburg" effect of aerobic glycolysis [19]. Altered mitochondrial function can drive tumor growth, mitochondria may be affected by oncogenic mutations, and they respond to metabolic stress. Mitochondria are therefore considered potential targets for cancer therapy [20]. Mitochondrial function and glucose metabolism vary between cancer types, as shown by differences in uptake of labelled glucose in PET scanning [21]. When mitochondria respond to metabolic demands and stress, they show morphological changes by undergoing fusion or fission $[19,20]$. Long, slender mitochondria are associated with a high rate of oxidative phosphorylation, but shorter mitochondria have been linked to reduced rate [22]. Damage to the inner membrane structure leads to failure of oxidative phosphorylation and reduced flux through the TCA cycle. The morphological differences observed between the two cell lines and PA-treated vs. control cells are therefore in line with the observed metabolic changes. Thus, T-47D had shorter mitochondria than AsPC-1 at baseline and lower rate of oxidative phosphorylation and following exposure to PA both cell lines showed morphological changes that are associated with the measured decrease in oxidative phosphorylation and reduced flux through the TCA cycle.

We have previously reported the anti-proliferative effects of PA against several cancer cell lines of different origin $[4,9,10]$. The current study shows how PA is metabolically processed and expelled from the cell and indicates that the final cellular response to PA depends on the metabolic state and mitochondrial function of the cell. The ability of PA to inhibit proliferation of several different types of cancer cells makes it a promising candidate for further drug development. Such further studies should take into consideration that the response will be context-dependent and that cancer cells with reduced mitochondrial respiration might be particularly susceptible.

\section{Material and Methods}

\section{Isolation of protolichesterinic acid}

The lichen $C$. islandica was collected in southwest Iceland [Latitude: 63.90744978, Longitude: - 22.44302969] on July 8th, 2013. The collection site is an open area, not privately owned. The lichen material was morphologically identified by Dr. Starri Heidmarsson, lichenologist. A voucher specimen (herbarium number LA-31883) is deposited at the Icelandic Natural History Museum, Akureyri. Isolation and identification of [+]-protolichesterinic acid (PA) was performed as described [8] to over $98 \%$ purity assessed by HPLC-UV. The structure of PA was further confirmed by UPLC-Quadrupole Time of Flight Mass Spectrometer [Waters Synapt G1 Mass Spectrometer, Waters, Manchester, UK; Fig. 2S, Supporting Information). PA was dissolved in ethanol absolute $\geq 99.8 \%$ (Sigma-Aldrich) and diluted for use in tissue-culture medium. All tests include controls in which the equivalent concentration of ethanol was used, up to $5 \mu \mathrm{L} / \mathrm{mL}$.

\section{Cell culture}

Cell lines were obtained from the American Type Culture Collection (ATTC) through LGC Promochem. AsPC-1 (pancreatic carcinoma cell line) and T-47D (breast ductal carcinoma cell line) were grown adherent. Both cell lines were cultured in RPMI-1640 tissue culture medium (GIBCO), containing penicillin $(50 \mathrm{IU} / \mathrm{mL})$ and streptomycin $(50 \mu \mathrm{g} / \mathrm{mL})$ (Invitrogen). Cells were cultured with fetal bovine serum ([FBS, GIBCOTM) to a final concentration of $10 \%$, and T-47D cells with an addition of $0.01 \mathrm{mg} / \mathrm{mL}$ insulin (Sigma). AsPC-1 cells were used between passages 2-32, and T47D cells between passages $2-26$. Both cell lines were maintained in a humidified incubator at $37^{\circ} \mathrm{C}$ and $5 \% \mathrm{CO}_{2}$ and sub-cultured following detachment by trypsin (0.25\% Trypsin/EDTA, Difco) as appropriate [8].

\section{Transmission electron microscopy}

Cells were seeded at $2 \times 10^{4}$ cells per well, grown as monolayers on glass coverslips (12 mm, size 1, Heinz Herenz) until 80-90\% confluence in 24-well plates (Corning Incorporated) and prepared for electron microscopy. The medium was removed, and cells were fixed with $2 \%$ glutaraldehyde (Ted Pella, Inc.) in $0.1 \mathrm{M}$ Cacodylate buffer ( $\mathrm{pH} 7.4$, J. B. EM Services Inc.) for 20 minutes. The fixative was removed, and cells washed in $0.1 \mathrm{M}$ Cacodylate buffer twice for 3 minutes. Coverslips were postfixed in 1\% osmium tetroxide (J.B. EM Services Inc.) in $0.1 \mathrm{M}$ Cacodylate buffer for 1 hour, followed by two 3-minute washes with $0.1 \mathrm{M}$ Cacodylate buffer. Cells were dehydrated in a series of ethanol: once with $70 \%$ ethanol (Gamla Apótekið) for 1 minute, once with $96 \%$ ethanol for 1 minute and twice with absolute ethanol for 1 minute. Each coverslip was then dipped in acetone for a few seconds and placed on an aluminium dish (Sigma-Aldrich). A drop of resin (Spurr Resin - Ted Pella, Inc.) was immediately poured on top of the coverslip to cover the cells. A gelatin capsule was filled with resin and inverted on top of the cells to create a block. Samples were incubated for 2 hours at room temperature to allow the resin to infiltrate into the cells. Blocks were then transferred to a $70^{\circ} \mathrm{C}$ oven and baked overnight. After cooling, resin blocks were 
separated from coverslips by dipping them for few seconds into liquid nitrogen. Blocks were trimmed with trimming tool (Leica EM Trim 2) and ultra-thin (100 nm) sections cut with a diamond knife ( $45^{\circ}$ Diatome) on an Ultramicrotome (Leica EM UC7) and placed on copper grids (Ted Pella, Inc.). Sections on grids were imaged using a JEM-1400PLUS PL Transmission Electron Microscope.

\section{Metabolomics}

For measurement of specific metabolites, cells were seeded in 6-well plates (Corning Incorporated) at 30000 cells per well and grown until 70-80\% confluency. Cells were treated with $5 \mu \mathrm{g} / \mathrm{mL}$ PA and appropriate controls for 24 hours. Supernatant media were collected, and the cells were washed three times with phosphate-buffered saline. Thirty microliters of an isotopically labelled internal standard mixture were added to each well, followed by $1 \mathrm{~mL}$ of ice-cold $80 \%$ methanol. The cells were dislodged with a cell scraper, and the cell extracts were collected into Eppendorf tubes. An additional $0.5 \mathrm{~mL}$ of $80 \%$ methanol was added to each well, and the resulting solutions, with any remaining cell debris, were combined with the previous extracts. The cell extracts were centrifuged $\left(20817 \times \mathrm{g}, 4^{\circ} \mathrm{C}\right)$ for 15 minutes to separate the supernatant from the precipitate. The supernatants were collected into new tubes, evaporated until dry using a vacuum concentrator (MiVac, Genevac Ltd., Ipswitch, England), and reconstituted in $300 \mu \mathrm{L}$ of acetonitrile/water $(1: 1)$. Prior to analysis, the samples were filtered by centrifugation with Pierce protein 96 well precipitation plate $\left(2000 \times \mathrm{g}, 4^{\circ} \mathrm{C}\right.$ for $\left.30 \mathrm{~min}\right)$.

For the extraction of extracellular metabolites, $30 \mu \mathrm{L}$ of an isotopically labelled internal standard mixture were added to $200 \mu \mathrm{L}$ of the spent medium from each sample. Five-hundred microliters of cold methanol were added to each sample, the samples vortexed for 1 minute, and centrifuged $\left(20817 \times \mathrm{g}, 4^{\circ} \mathrm{C}\right)$ for $15 \mathrm{~min}$ utes to pellet the precipitate. The supernatants were collected into new tubes and dried using a vacuum concentrator (MiVac, Genevac Ltd., Ipswitch, England). Finally, the samples were reconstituted in $600 \mu \mathrm{L}$ acetonitrile/water $(1: 1)$ and filtered by centrifugation with Pierce protein 96 -well precipitation plate $(2000 \times \mathrm{g}$, $4^{\circ} \mathrm{C}$ for $\left.30 \mathrm{~min}\right)$.

Intra- and extracellular samples were analysed by hydrophilic interaction liquid chromatography [HILIC] coupled with time-offlight (TOF) mass spectrometry (Synapt G2, Waters, UK) using a previously described method [23]. Each sample was measured both in positive and negative ionization mode. The mass spectrometer (MS) was operated using a capillary voltage of $1.5 \mathrm{kV}$, sampling cone voltage of $30 \mathrm{~V}$, and extraction cone voltage of $4 \mathrm{~V}$. The chromatographic separation was achieved by using an Acquity BEH amide $1.7 \mu \mathrm{m}$ column $(2.1 \times 150 \mathrm{~mm}$; Waters $)$, a flow rate of $0.4 \mathrm{~mL} / \mathrm{min}$, column temperature of $45^{\circ} \mathrm{C}$, and an injection volume of $5 \mu \mathrm{L}$. Mobile phase A consisted of ACN containing $0.1 \%$ $(\mathrm{v} / \mathrm{v})$ formic acid, and mobile phase $\mathrm{B}$ consisted of $\mathrm{H}_{2} \mathrm{O}$ containing $0.1 \%(v / v)$ formic acid. The following elution gradient was used: 0 minutes $99 \%$ A, 6 minutes $40 \%$ A, 8 minutes $60 \%$ A, 8.5 minutes $99 \% \mathrm{~A}$, and 14 minutes 99\% A. Quadrupole-TOF tandem mass spectrometry was used to analyze selected ions in both positive and negative ionization modes using the same chromatographic conditions and mass spectrometer configurations described above. The raw data files were converted to netCDF files using the software Databridge 1.0 (Waters, UK). The R package XCMS [12] was used for automated feature detection and retention time alignment using the Centwave and Obiwrap algorithms, respectively. Metabolites were annotated by comparing $\mathrm{m} / \mathrm{z}$ and retention times to an in-house library. The identified metabolites were normalized by dividing the chromatographic peak area of each metabolite with the peak area of a proper internal standard.

\section{Real-time ATP rate assay}

To assess general mitochondrial function, the Agilent Seahorse XFp Real-Time ATP Rate Assay was used. The instrument, all consumables, and analysis programs were obtained from Seahorse Bioscience [Agilent], MA, USA. Cells were seeded into 96-well Seahorse XFp assay plates at different densities from $1 \times 10^{4}$ to $4 \times 10^{4}$ per well in normal growth media [see Cell Culture]. Cells were either pretreated with $5 \mathrm{\mu g} / \mathrm{mL}$ of PA and solvent control for $24 \mathrm{~h}$ or treatment was added during the assay run. On the day of assay, cell medium was exchanged for assay test medium (RPMI with $1.0 \mathrm{M}$ glucose, $100 \mathrm{mM}$ sodium pyruvate, and $200 \mathrm{nM}$ L-glutamine) and placed at $37^{\circ} \mathrm{C}$ in a non-CO2 incubator for $1 \mathrm{~h}$ before conducting assays in the NADP/NADOH Seahorse XFp Analyzer. The Real-Time ATP Rate Assay involved sequential injections of PA $(5 \mu \mathrm{g} / \mathrm{mL})$, solvent or clean medium in the first port, oligomycin $[1,5 \mu \mathrm{M}]$, and Rotenone/antimycin $\mathrm{A}([0,5 \mu \mathrm{M})$ in next two. Data were analyzed using the Seahorse software Wave 2.6.0.

\section{Glucose metabolism}

For measurements of glucose and lactate in culture supernatants, cells were seeded in 6-well plates (Corning Incorporated) at $3 \times 10^{4}$ per well and grown until $70-80 \%$ confluence. Subsequently, cells were treated with $5 \mu \mathrm{g} / \mathrm{mL}$ PA and appropriate controls for 24 hours. $0.5 \mathrm{~mL}$ of the medium was collected from each well as well as a clean medium, medium containing $5 \mu \mathrm{g} / \mathrm{mL}$ PA and solvent as controls. Samples were measured in Radiometer ABL800 Flex (Radiometer Medical ApS) and results exported to Excel spreadsheet.

\section{Assessment of redox status and reactive oxygen species - ROS}

For all experiments cells were seeded in white flat-bottom 96-well plates (BRANDplates, BRAND GMBH + CO KG) at $5 \times 10^{3}-1 \times 10^{4}$ cells per well and grown for 24 hours. Cells were then treated with $5 \mathrm{\mu g} / \mathrm{mL}$ PA and appropriate controls for 24 hours.

Changes in total and oxidized glutathione were measured by using the GSH/GSSG-Glo Assay kit (cat. No. V6611, Promega) according to the manufacturer's instructions. A NADP/NADPH-Glo Assays kit (cat. No. G9081, Promega) was used according to the manufacturer's instructions to detect total oxidized and reduced nicotinamide adenine dinucleotide phosphates [NADP+ and $\mathrm{NADPH}$ ] and determine their ratio. $\mathrm{H}_{2} \mathrm{O}_{2}$ was measured by using the ROS-Glo $\mathrm{H}_{2} \mathrm{O}_{2}$ Assay kit (cat. no. G8820; Promega) according to the manufacturer's instructions. $\mathrm{H}_{2} \mathrm{O}_{2}$ substrate solution was added for $2 \mathrm{~h}$ before the luminescence reading for optimal results. Menadione (Sigma) was used as optimal positive control for $\mathrm{H}_{2} \mathrm{O}_{2}$ induction. For AsPC-1 cells $50 \mu \mathrm{M}$ menadione was added for $2 \mathrm{~h}$ and for T-47D cell $100 \mu \mathrm{M}$ menadione was added for $24 \mathrm{~h}$ to obtain positive response. 
All assays are based on a luminescent signal generated by a chemical reaction appropriate for each assay. The luminescence was measured using (Modulus II Microplate Multimode Reader, Turner BioSystems) in integration time of 0.5 second per well and results exported to Excel spreadsheet. To account for differences in cell number or protein amount between experiments, signal intensities were normalized to the mean signal intensity within each batch measurement to allow comparison between batches.

\section{Statistical analysis}

Principal components analysis (PCA), Welch's t-test, and the Bonferroni correction for multiple comparisons were performed using R v3.5.1 (R Development Core Team, Vienna, Austria).

\section{Supporting information}

Supplementary Figure 1: Glucose uptake and lactate secretion. Supplementary Figure 2: Mass spectra and molecular structure of protolichesterinic acid (PA) and PA conjugates measured with qTOF tandem mass spectrometry. Supplementary Figure 3: Changes to derivatives of lysophosphatidyl choline and carnitine. Supplementary Figure 4: Trends towards lowered ROS following PA treatment.

\section{Contributors' Statement}

FJ performed and analyzed the metabolomics. PC performed all experiments and TEM analysis, MX isolated, purified and characterized protolichesterinic acid, ÓR analyzed and interpreted metabolomics and Sea Horse data, HMÖ designed the study and interpreted all data. All authors contributed to the writing of the manuscript.

\section{Acknowledgements}

The financial support of the University of Iceland Research Fund (1238-123380) is gratefully acknowledged.

\section{Conflict of Interest}

The authors declare that they have no conflict of interest.

\section{References}

[1] Xu M, Heidmarsson S, Thorsteinsdottir M, Kreuzer M, Hawkins J, Omarsdottir S, Olafsdottir ES. Authentification of Iceland Moss [Cetraria islandica] by UPLC-QToF-MS chemical profiling and DNA barcoding. Food Chem 2018; 245: 989-996

[2] Xu M, Heidmarsson S, Olafsdottir ES, Buonfiglio R, Kogej T, Omarsdottir S. Secondary metabolites from cetrarioid lichens: Chemotaxonomy, biological activities and pharmaceutical potential. Phytomedicine 2016; 23: 441-459

[3] Ogmundsdóttir HM, Zoëga GM, Gissurarson SR, Ingólfsdóttir K. Antiproliferative effects of lichen-derived inhibitors of 5-lipoxygenase on malignant cell-lines and mitogen-stimulated lymphocytes. J Pharm Pharmacol 1998; 50: 107-115

[4] Haraldsdóttir S, Gudlaugsdóttir E, Ingólfsdóttir K, Ogmundsdóttir HM. Anti-proliferative effects of lichen-derived lipoxygenase inhibitors on twelve human cancer cell lines of different tissue origin in vitro. Planta Med 2004; 70: 1098-1100
[5] Brisdelli F, Perilli M, Sellitri D, Piovano M, Garbarino JA, Nicoletti M, Bozzi A, Amicosante G, Celenza G. Cytotoxic activity and antioxidant capacity of purified lichen metabolites: an in vitro study. Phytother Res 2013; 27: 431-437

[6] Ingolfsdottir K, Breu W, Huneck S, Gudjonsdottir GA, Müller-Jakic B, Wagner $\mathrm{H}$. In vitro inhibition of 5 -lipoxygenase by protolichesterinic acid from Cetraria islandica. Int Immunopharmacol I 1994; 1: 187-191

[7] Bucar F, Schneider I, Ogmundsdóttir H, Ingólfsdóttir K. Anti-proliferative lichen compounds with inhibitory activity on 12[S]-HETE production in human platelets. Int Immunopharmacol 2004; 11: 602-606

[8] Pidgeon GP, Lysaght J, Krishnamoorthy S, Reynolds JV, O'Byrne K, Nie D, Honn KV. Lipoxygenase metabolism: roles in tumor progression and survival. Cancer Metastasis Rev 2007; 26: 503-524

[9] Bessadóttir M, Eiríksson FF, Becker S, Ögmundsdóttir MH, Ómarsdóttir S, Thorsteinsdóttir M, Ögmundsdóttir HM. Anti-proliferative and proapoptotic effects of lichen-derived compound protolichesterinic acid are not mediated by its lipoxygenase-inhibitory activity. Prostaglandins Leukot Essent Fatty Acids 2015; 98: 39-47

[10] Bessadóttir M, Skúladóttir E, Gowan S, Eccles S, Ögmundsdóttir S, Ogmundsdóttir HM. Effects of anti-proliferative lichen metabolite, protolichesterinic acid on fatty acid synthase, cell signalling and drug response in breast cancer cells. Phytomedicine 2014; 21: 1717-1724

[11] Pengsuparp T, Cai L, Constant H, Fong HH, Lin LZ, Kinghorn AD, Pezzuto JM, Cordell GA, Ingolfsdóttir K, Wagner H. Mechanistic evaluation of new plant-derived compounds that inhibit HIV-1 reverse transcriptase. J Nat Prod 1995; 58: 1024-1031

[12] Brinkman K, Kakuda TN. Mitochondrial toxicity of nucleoside analogue reverse transcriptase inhibitors: a looming obstacle for long-term antiretroviral therapy? Curr Opin Infect Dis 2000; 13: 5-11

[13] Smith CA, Want E], O'Maille G, Abagyan R, Siuzdak G. XCMS: processing mass spectrometry data for metabolite profiling using nonlinear peak alignment, matching, and identification. Anal Chem 2006; 78: 779-787

[14] Elia I, Broekaert D, Christen S, Boon R, Radaelli E, Orth MF, Verfaillie C, Grünewald TGP, Fendt SM. Proline metabolism supports metastasis formation and could be inhibited to selectively target metastasizing cancer cells. Nat Commun 2017; 8: 15267

[15] Gunawardena G. Michael Addition. Accessed February 1, 2021 at: https://chem.libretexts.org/Ancillary_Materials/Reference/Organic_ Chemistry_Glossary/Michael_Addition

[16] Punganuru SR, Madala HR, Arutla V, Srivenugopal KS. Selective killing of human breast cancer cells by the styryl lactone [R]-goniothalamin is mediated by glutathione conjugation, induction of oxidative stress and marked reactivation of the $\mathrm{R} 175 \mathrm{H}$ mutant $\mathrm{p} ; 53$ protein. Carcinogenesis 2018; 39: 1399-1410

[17] Lu SC. Glutathione synthesis. Biochim Biophys Acta 2013; 1830: 31433153

[18] Le Lamer AC, Authier H, Rouaud I, Coste A, Boustie J, Pipy B et al. Protolichesterinic acid derivatives: $\alpha$-methylene- $\gamma$-lactones as potent dual activators of PPARy and Nrf2 transcriptional factors. Bioorg Med Chem Lett 2014; 24: 3819-3822

[19] Bensinger S], Christofk HR. New aspects of the Warburg effect in cancer cell biology. Semin Cell Dev Biol 2012; 23: 352-361

[20] Grasso D, Zampieri LX, Capelôa T, Van de Velde JA, Sonveaux P. Mitochondria in cancer. Cell Stress 2020; 4: 114-146

[21] Lee ST, Lawrentschuk N, Scott AM. PET in prostate and bladder tumors. Semin Nucl Med 2012; 42: 231-246

[22] Picard M, Shirihai OS, Gentil B], Burelle Y. Mitochondrial morphology transitions and functions: implications for retrograde signaling? Am J Physiol Regul Integr Comp Physiol 2013; 304: R393-R406

[23] Jóhannsson F, Árnason N, Landrö R, Guðmundsson S, Sigurjonsson ÓE, Rolfsson Ó. Metabolomics study of platelet concentrates photochemically treated with amotosalen and UVA light for pathogen inactivation. Transfusion 2020; 60: 367-377 